\title{
Power Quality Disturbance Detection Based on Mathematical Morphology and Fractal Technique
}

\author{
Gengyin Li, Member, IEEE, Ming Zhou, Student Member, IEEE, \\ Yan Luo, and Yixin Ni, Senior Member, IEEE
}

\begin{abstract}
Based on mathematical morphology and grille fractal, a novel approach on power quality disturbance detection and location is presented in this paper. At first a parallel composite morphological filter with multiple-structure elements is designed to filter the random noise and impulse noise in power quality disturbance signals. Then to the filtered curves, an easy implementation criterion for singularity detection based on the change regularity of grille fractal is proposed to locate the start and end time that disturbance occurs. The voltage sag, swell, harmonic and their combined disturbances are used to verify the validity of the proposed filter-location approach. Numerical results show that the proposed approach is valid and effective.
\end{abstract}

Index Terms-- dynamic power quality; disturbance detection; mathematical morphology; morphologic filter; grille fractal

\section{INTRODUCTION}

$\mathrm{P}$ ower quality disturbance detection has been a research hot point in the area of power quality analysis. In actual processing, the sampling signal is often mixed with different kinds of noise due to sampling errors, random disturbances and other unstable factors. The random noise is also called as singular point. When there exists great deal of noise mixed with disturbance singular points in the sampling signal, it may bring large errors when distinguishing the singular points caused by power quality disturbances. How to extract transient information from sampling signals with random noise has vital role in analyzing power quality disturbances.

There are several approaches on this problem. Wavelet Transform (WT) has been proved effectively in signal filtering [1], but due to its large calculation amount and difficulty of implementation by cheap hardware, it is not suitable for realtime application. In contrast with wavelet analysis, Mathematical Morphology (MM) is developed from set theory and integral geometry, and is concerned with the shape of a signal waveform in the complete time domain rather than the frequency domain [2]. MM has been widely applied in the

This work was supported in part by Specialized Research Fund for the Doctoral Program of Higher Education, under Ministry of Education of the People's Republic of China (No. 20040079002).

Gengyin Li, Ming Zhou and Yan Luo are with the Key Laboratory of Power System Protection and Dynamic Security Monitoring and Control under Ministry of Education, North China Electric Power University, Baoding 071003, Hebei Province, P.R.China (e-mail: ligy@ncepu.edu.cn, zhouming@ncepu.edu.cn, lucyluoncepu@yahoo.com.cn).

Yixin Ni is with the Department of Electrical and Electronic Engineering, The University of Hong Kong, Hong Kong SAR, P.R.China. (e-mail: yxni@eee.hku.hk) areas of image processing, machine vision, and pattern recognition [3]-[7], due to its robustness in preserving the shape while suppressing noise. MM can be developed as an efficient nonlinear filter. References [6], [7] have presented a morphological filter with the average combination of openingclosing and closing-opening filtering, to filter white noise and impulse noise, respectively. Using morphological operators, the radical shape of the disturbed signal can be recognized, reconstructed and enhanced, even if the original signal is mixed with strong noise or serious distortion. In addition, MM has the feature of easy calculation and implementation.

Singularity is an important feature of disturbance signal, which is also an important gist of classification and recognition to disturbances. In order to exactly identify the singularity of disturbed signals, researching its local property is required. In a long period, Fourier Transform (FT) is a main tool of studying the singularity of signals. By studying the decaying speed of the function in FT field, the function whether has singularity or the size of its singularity is concluded. However, FT can only determine the whole property of the signal while it is short in placing local property. So FT is difficult for determining the position and distribution singular points in space. While WT, which possesses capabilities of time and frequency domain localizations, gives a great impetus in signal singularity detection. However, how to select an appropriate mother wavelet is a difficult task since improper mother wavelet may reduce the detection effect. Fractal geometry is the generic terms of self-similar figure and structure in certain meaning, which have not feature length. In recent years, fractal technique has been applied to fault analysis, radar signal detection, etc., with favorable effects [9]-[12].

Based on the MM and parallel composite filters with multiple Structuring Elements (SEs), this paper constitutes a comprehensive filter as the pre-unit for detecting disturbance. This filter can filter several kinds of noise at one time. Then according to the algorithm of grille fractal dimension, a new method using its grille change regularity is proposed to detect singular points, which can locate the start and end time of power quality disturbances effectively and rapidly. The proposed morphology-grille algorithm is with relatively easier, smaller computing time and preferable utility.

\section{Mathematical Morphology-BASEd Signal FiLtering}

\section{A. Mathematical Morphology}

Based on the research achievement of integral geometry, G.Matheron and J.Serra founded MM theory in 1964 [16]. Its 
basic idea is to use a probe, which is named as SE, to collect information of the signals. As the SE moving in the signal constantly, it can review the interrelation among every part, and pick up useful information to analyze and describe the signals. Morphological transform can decompose a complicated signal into several parts that have different physical significance. It can pick up the signal from the background and keep its main shape trait at the same time. Using MM to construct a low band filter, even if the original signal is mixed with large amount of noise, its radical shape can still be recognized, reconstructed and enhanced. So, using the MM to smooth, filter and enhance the helpful signal is propitious to the following signal singular analysis and disturbance classification.

In MM, two basic morphological operators are erosion and dilation. Based on these two operators, several common compound operators, opening, closing, Hit-Miss and Top-Hat transform, so on, are defined [12]. Morphological transform is usually classified into two-valued and $n$-valued transform. $\mathrm{N}$ valued transform is also called grey transform. Because the power system signal analysis commonly deals with onedimensional signal, this paper only introduces two-valued transform, which includes erosion, dilation, opening, closing and the compound opening and closing, under the onedimensional discrete condition.

Suppose that the definition domain of the input signal $f(\mathrm{n})$ and the sequential SEs $g(m)$ is $D_{f}=\left\{x_{1}, x_{2}, \ldots, x_{N}\right\}$ and $D_{g}=\left\{y_{1}\right.$, $\left.y_{2}, \ldots, y_{M}\right\}$, respectively, and $N>M$.

The erosion of $f(n)$ by $g(m)$, denoted as $f \ominus g$, is defined as

$(f \ominus g)(n)=\min \left\{f(n+m)-g(m) \mid(n+m) \in D_{f}, m \in D_{g}\right\}$

The dilation of $f(n)$ by $g(m)$, denoted as $f \ominus g$, is defined by

$(f \oplus g)(n)=\max \left\{f(n-m)+g(m) \mid(n-m) \in D_{f}, m \in D_{g}\right\}$

Erosion is a kind of shrinking transform, which can make the target signal contract while holes enlarging. Dually, dilation is an expanding process, which realizes the target signal enlarging together with holes contracting. Generally, erosion and dilation are not reversible each other. So the conjugation of them can form new morphological operators, named by opening and closing operators, which are also important operators in MM, defined as follows.

The opening of $f(n)$ by $g(m)$, denoted as $f \circ g$, is defined by

$$
(f \circ g)(n)=[(f \ominus g) \oplus g](n)
$$

The closing of $f(n)$ by $g(m)$, denoted as $f \bullet g$, is defined by

$$
(f \bullet g)(n)=[(f \oplus g) \ominus g](n)
$$

Generally speaking, opening operation is used to eliminate scrap points, sparks and "little bridges", or say smoothing the image. And closing operator is used to stuff "little holes" or connect the contiguous two regions. In actual application, to one-dimension signal, opening operation is mainly used to smooth and restrain peak noise. Closing operation is chiefly used to restrain wave valley noise. Maragos utilized the same SEs to compose the opening-closing (OC) and closingopening (CO) filters through proper calculation combination, which can restrain both positive and negative impulse noise
[3], [4], defined as

$$
\begin{aligned}
& O C[f(n)]=(f \circ g \bullet g)(n) \\
& C O[f(n)]=(f \bullet g \circ g)(n)
\end{aligned}
$$

$\mathrm{OC}$ and $\mathrm{CO}$ filters have all the features of opening and closing operation. Although they can filter positive and impulse noise together, statistic bias is existed. Because of opening operator's expansibility and closing operator's contractibility, the output magnitude of the OC filter becomes small. On the contrary, the output magnitude of the $\mathrm{CO}$ filter is large. So there will not get a good filtering result if using anyone alone. However, using the average value of these two filters can approach the original signal very well. This kind of combination filter is widely used in image process and signal process [3], [4].

Suppose that the input signal is $f(n)$, which is composed by the original signal $x(n)$ and the noise signal $s(n)$.

$$
f(n)=x(n)+s(n), n=1, \ldots, N
$$

Then the output signal of the composite filter is

$$
y(n)=\frac{1}{2}\{O C[f(n)]+C O[f(n)]\}
$$

\section{B. Parallel Composite Filters with Multi-SE}

The output of morphological filter is not only determined by the transform modality, but also lies on the size and shape of the SE. Commonly, only when is the signal suited for the size and shape of the SE, it can be well preserved. Experience shows that semicircular SE is better to filter white noise, and its radius should be within 2 to 10 percent of the magnitude of the pending filtering waveform. And more smaller the radius is, more higher the precision of the filtering is. The length of the SE should not exceed by 25 percent of the sampling-point number. To the impulse noise, trigonal SE is generally adopted. If the length of the longest impulse noise is $T$, the sampling period is $T s$, theoretically speaking, the length of the SE $M$ should bigger than $T / T s$.

Despite the $\mathrm{OC}$ and $\mathrm{CO}$ filters can filter positive and negative impulse noise simultaneously, they only have better filter results to certain specific noise, because they only use a kind of SE. For example, it will get better filter result to white noise if using semicircular SE in signal filtering. Only adopting one SE will not get the best filter effect in cases where there are more than one kind of noise in the signal.

In order to improve existing methods, this paper constructs a kind of parallel composite filter with multi-SE. This kind of filter not only can restrain many kinds of noise effectively but also keeps more useful information. Here we call them the OC maximal and $\mathrm{CO}$ minimal filter (OCCO filter).

Suppose that there is an input signal $f(n)$ and a sequential multiple SE aggregation $\left\{g_{1}\left(m_{1}\right), g_{2}\left(m_{2}\right), \ldots, g_{l}\left(m_{l}\right)\right\}$. The definitions of the OCCO filter are

$$
\begin{aligned}
& \psi_{o c}[f(n)]=\operatorname{MAX}\left(O C g_{1}, O C g_{2}, \cdots, O C g_{l}\right) \\
& \psi_{c o}[f(n)]=\operatorname{MIN}\left(C O g_{1}, C O g_{2}, \cdots, C O g_{l}\right)
\end{aligned}
$$

where, $O C g_{i}=\left(f \circ g_{i} \bullet g_{i}\right)(n)(i=1,2, \cdots, l)$

$$
C O g_{i}=\left(f \bullet g_{i} \circ g_{i}\right)(n)(i=1,2, \cdots, l)
$$

Because the output of the OC filter is smaller and the 
output of the CO filter is larger than the original signal, we take the maximum output of the $\mathrm{OC}$ filter and the minimum outcome of the CO filter in multiple SE filtering, then take the average value of both outputs, this result could be much approach the practical signal. The framework of the OCCO filter is shown in Fig.1.

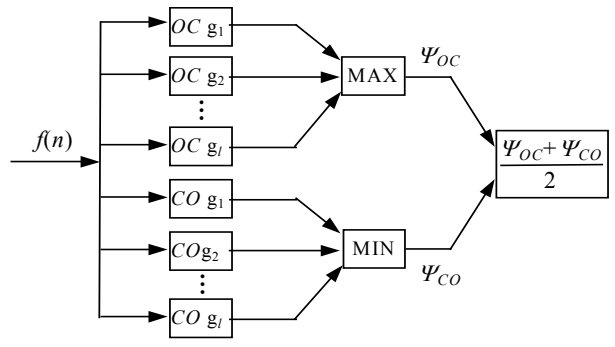

Fig. 1. Framework of the OCCO filter

Now use a numerical example to verify the proposed filter. Construct a signal with white noise of variance 0.1 and a pulse with the magnitude $1.5 \mathrm{pu}$. Now sampling frequency is $6.4 \mathrm{kHz}$ that is 128 sampling points per period. The adopted semicircular SE with the length 9 and radius 0.06 , is [0, $0.0397,0.052,0.058,0.06,0.058,0.052,0.0397,0]$. While the trigonal SE with the length 15 , is $[0,0.0214,0.0429,0.0643$, $0.0857,0.1071,0.1286,0.15,0.1286,0.1071,0.0857,0.0643$, $0.0429,0.0214,0]$. Use these two SEs to filter the signal respectively, and a part of the curve involved the pulse is shown in Fig.2.

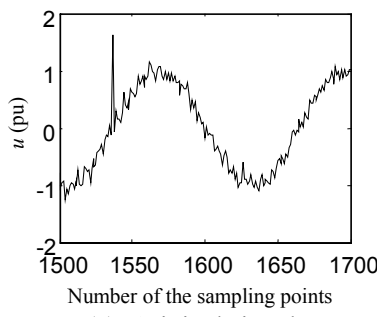

(a) Original signal

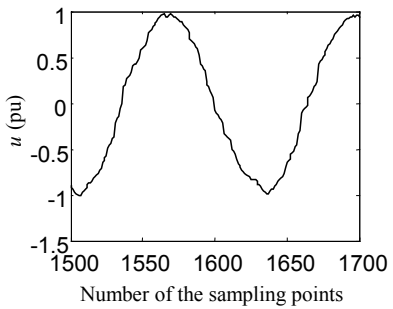

(c) Trigonal SE

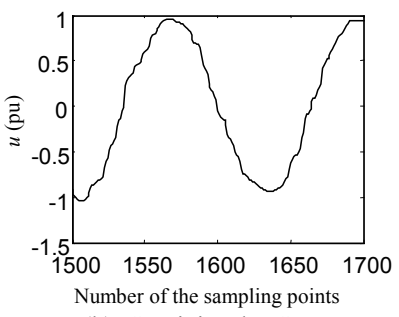

(b) Semicircular SE

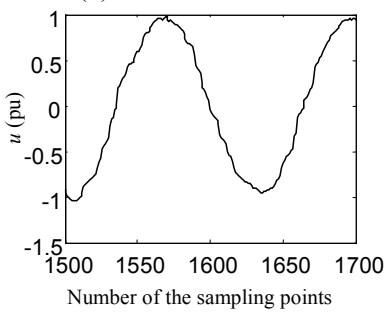

(d) Multi-SE
Fig.2. Comparison of filtering performance by using different SEs

Fig.2 shows that although the semicircular SE can also filter the pulse, the waveform, where the pulse happened, is not smoother than the one filtered by trigonal SE. Nevertheless, other parts in the waveform filtered by the semicircular element are much more smoother than the ones filtered by trigonal SE, especially at the peaks and valleys of the wave. The waveform filtered by both SEs has overcome these shortcomings. The result of the multi-SE filter is better, see (c) in Fig.2. The signal-to-noise ratio (SNR) of the original signal is $16.93 \mathrm{~dB}$, when the signal is filtered by the SEs, it becomes $24.20 \mathrm{~dB}, 24.29 \mathrm{~dB}$ and 24.41 , corresponding to the result of semicircular, trigonal and composite SE, respectively. Table I shows the comparison result.

Furthermore, simulation results show that, when there are more pulses, the multi-SE filter could achieve better results.

TABLE I

SNR COMPARISONS OF THREE FILTERS UNDER DifFERENT PULSE NumBERS (dB)

\begin{tabular}{ccccc}
\hline $\begin{array}{c}\text { Pulse } \\
\text { Number }\end{array}$ & $\begin{array}{c}\text { Signal } \\
\text { Siginal }\end{array}$ & $\begin{array}{c}\text { Signal after using } \\
\text { semicircular SE }\end{array}$ & $\begin{array}{c}\text { Signal after using } \\
\text { trigonal SE }\end{array}$ & $\begin{array}{c}\text { Signal after using } \\
\text { multi-SE }\end{array}$ \\
\hline 1 & 16.93 & 24.20 & 24.29 & 24.41 \\
3 & 16.54 & 24.04 & 23.93 & 24.18 \\
10 & 15.09 & 23.79 & 23.62 & 24.02 \\
\hline
\end{tabular}

\section{Signal Singularity Detection Based on Grill FRACTAL DEMENSION}

Mathematician Mandelbrot first proposed fractal geometry in 1980s [17]. Fractal geometry is the generic terms of selfsimilar figure and structure in certain meaning, which haven't feature length. It investigates the not smooth and irregular geometrical objectives in nature and nonlinear systems. Its fractal measure is fractal dimension. Fractal dimension reflects the complexity of the fractal set. More complex the fractal set is, much larger its fractal dimension will be. To the discrete digital signals, we can regard them as digital discrete space point sets. Usually, different signals have the different fractal dimensions. So fractal dimension can be used as signal detection [18]. The technique of fractal dimension has applied in high-impedance fault analysis of power systems with favorable effects [12].

There are many kinds of definitions to fractal dimension. To different fractal dimensions, the corresponding algorithms are different. Based on the definition of the grid in grille fractal dimension [14], [15], an easy realization approach is proposed in this paper. This approach can determine the start and recovery time of the disturbance occurrence accurately and rapidly.

Definition: the grille fractal dimension $N(\delta)$ is defined the number of the needed square grid with length of side $\delta$, covering the signal $u$ in interval $\left[t_{k}-\Delta t, t_{k}\right]$.

Suppose that the signal has $n+1$ ( $n$ is an even number) sampling points $\left(x_{1}{ }^{(k)}, x_{2}{ }^{(k)}, \ldots, x_{n+1}{ }^{(k)}\right)$ in interval $\left[t_{k}-\Delta t, t_{k}\right]$.

Assume $\delta=\Delta t / n$, then

$$
N(\delta)=\frac{1}{\delta} \sum_{j=1}^{n}\left|x_{j}-x_{j+1}\right|
$$

where $\delta$ is taken the time difference between two consecutive sampling points.

To a pure sinusoidal voltage wave, if $\Delta t$ is half period, then in any $\Delta t$ interval, $N(\delta)$ has the same value. If disturbance occurs, the signal waveform will change. Corresponding $N(\delta)$ will also change. In the actual processing of sampling, even if the signal has been filtered, it still cannot eliminate the affect of noise completely. There still exists little fluctuation in the curve $N$, for example shown in Fig.3.

According to the above analysis, we know that the grillechanging regularity is able to use detecting the start and end time. This paper adopts the algorithm, which compares the 


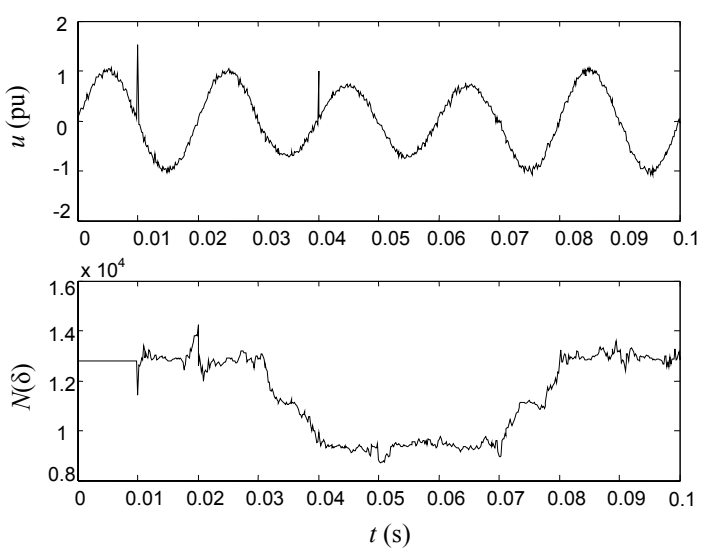

Fig. 3. Disturbance signal with noise and its grille-changing curve

grille number $N(\delta)$ at time $t$ with the average value of $N(\delta)$ before time $t$, to get the grille change curve, that is to check whether the $N(\delta)$ at time $t$ exceeds 20 percent of average $N(\delta)$ before time $t$. If it does, check whether each point in recent half period exceeds by 20 percent of average $N$. If they all do, then this point is regarded as a singular point, and denoting the detection factor $D_{m}=1$ at this point, otherwise $D_{m}=0$. When a singular point is found, the observation point jumps to the point after half period from the singular point, and the average value of $N$ will be recalculated from the new observation point.

Fig. 4 shows the result of time location with respect to no noise signal. In Fig.4, the preset start and end time of the disturbance are $0.03 \mathrm{~s}$ and $0.07 \mathrm{~s}$ respectively. The results given by the proposed algorithm are $0.0306 \mathrm{~s}$ and $0.0706 \mathrm{~s}$. The delays are $0.0006 \mathrm{~s}$ and $0.0006 \mathrm{~s}$, respectively. As above mentioned that even if the signal has been filtered, it still preserves the affect of noise. To those signals not filtered noise completely, this technique first gets the curve $N(\delta)$, then uses another filter with linear SEs to smooth the curve $N(\delta)$ to get a new curve $N$ '. The original signal in Fig.5 is a disturbed voltage one by noise. The disturbance time location is also shown in Fig.5, which is obtained after using the parallel composite filter with multi-SE introduced in section II. The simulated start and end time of the disturbance are $0.03 \mathrm{~s}$ and $0.07 \mathrm{~s}$. The results given by the approach are $0.0309 \mathrm{~s}$ and $0.0705 \mathrm{~s}$. The delays are $0.0009 \mathrm{~s}$ and $0.0005 \mathrm{~s}$.

Different from voltage sags and voltage swells, the value

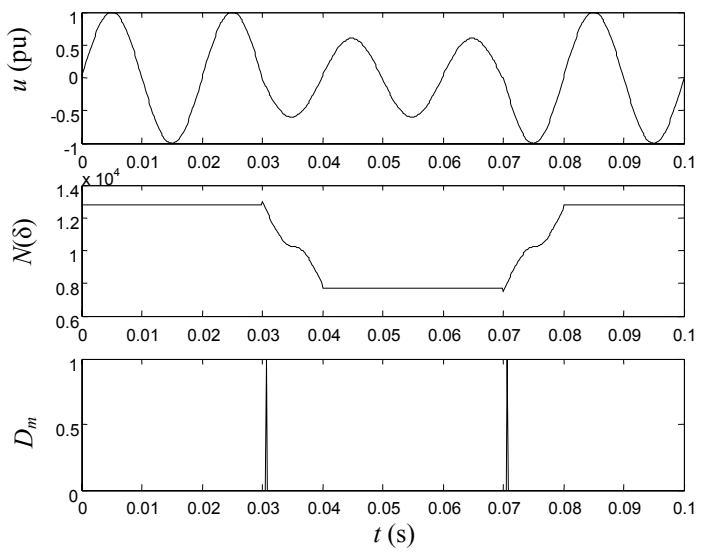

Fig. 4. Time location of disturbance signal without noise

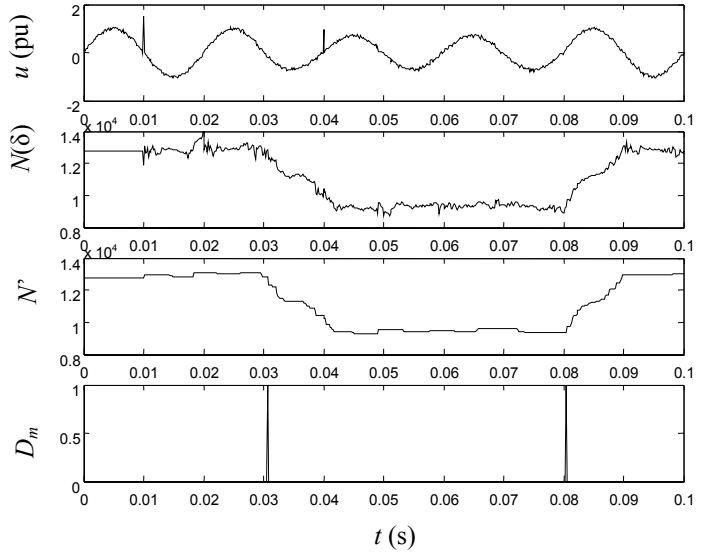

Fig. 5. Time location of disturbance signal with noise

$N(\delta)$ in the harmonic distortion signals changes continuously, unlike the $N(\delta)$ of former signals will reach a steady value after a short transition which is shown in Fig. 6. But we still can see the changing-regularity of the curve $N$. The analysis shows that if the curve $N(\delta)$ has been processed by the grillechanging algorithm once to get $N^{\prime \prime}$, further analyzing $N^{\prime \prime}$ can get the start and end time of harmonic distortion signals. In Fig. 6, the preset start and end time of the disturbance are $0.03 \mathrm{~s}$ and $0.11 \mathrm{~s}$. The final results are $0.0305 \mathrm{~s}$ and $0.1103 \mathrm{~s}$. The delay is $0.0005 \mathrm{~s}$ and $0.0003 \mathrm{~s}$, respectively.

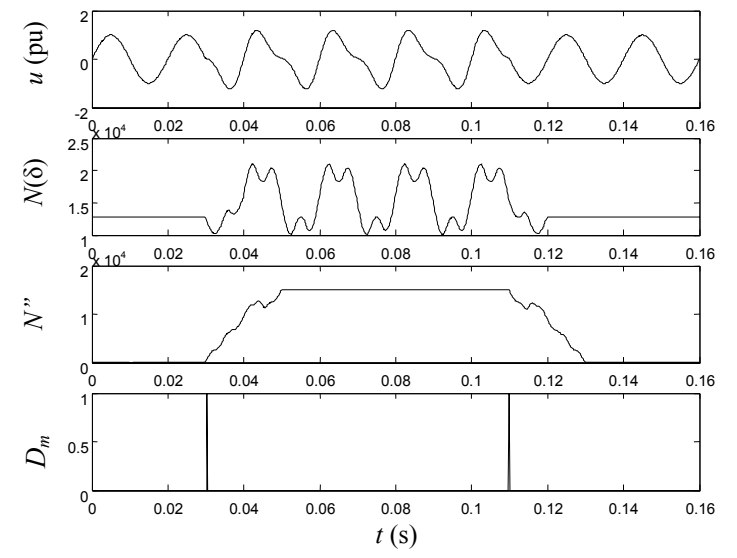

Fig. 6. Time location of harmonic distortion disturbance signal without noise

According to the above analysis, we know that using the grille algorithm defined in (11) can locate the disturbance time. If the signal with noise, it is necessary using a simple morphological method to smooth it before it is analyzed. If there is harmonic distortion, the grille analysis to the curve $N(\delta)$ is needed, then the disturbance time can be located.

\section{Simulation Results}

In order to verify the validity of the proposed filter algorithm, computer simulation analyses are undertaken. The sampling frequency to the original signal is 128 points per period. In this simulation, we use two semicircular SEs and two trigonal SEs. One of semicircular SEs with length 17 and radius 0.08 is $[0,0.0387,0.0529,0.0624,0.0693,0.0742$, $0.0775,0.0794,0.08,0.0794,0.0775,0.0742,0.0693,0.0624$, 
$0.0529,0.0387,0]$ (denoted by SE1). Another with length 13 and radius 0.06 is $[0,0.0332,0.0447,0.052,0.0566,0.0592$, $0.06,0.0592,0.0566,0.052,0.0447,0.0332,0]$ (SE2). One of the trigonal SEs with length is 13 , is $[0,0.0167,0.0333,0.05$, $0.0667,0.0833,0.1,0.0833,0.0667,0.05,0.0333,0.0167,0]$ (SE3). Another, whose length is 15 , is $[0,0.0214,0.0429$, $0.0643,0.0857,0.1071,0.1286,0.15,0.1286,0.1071,0.0857$, $0.0643,0.0429,0.0214,0]$ (SE4). Some kinds of disturbances and their simulation results are made as follows.

\section{A. Voltage Sags}

Voltage sags are referred to the magnitude of voltage under $0.9 \mathrm{pu}$ (in the range of $0.1 \sim 0.9 \mathrm{pu}$ ) lasting for $10 \mathrm{~ms} \sim 1 \mathrm{~min}$, which is generally caused by the electric motor startup or switching operation of power equipments. Voltage sags cause the equipment operating abnormally. Fig. 7 shows the location results for a disturbed voltage curve with a sag lasting for $50 \mathrm{~ms}$ and the falling magnitude by $30 \%$, and white noise variance 0.04 together with two pulses with magnitudes $1.5 \mathrm{pu}$ and $1 \mathrm{pu}$, respectively.

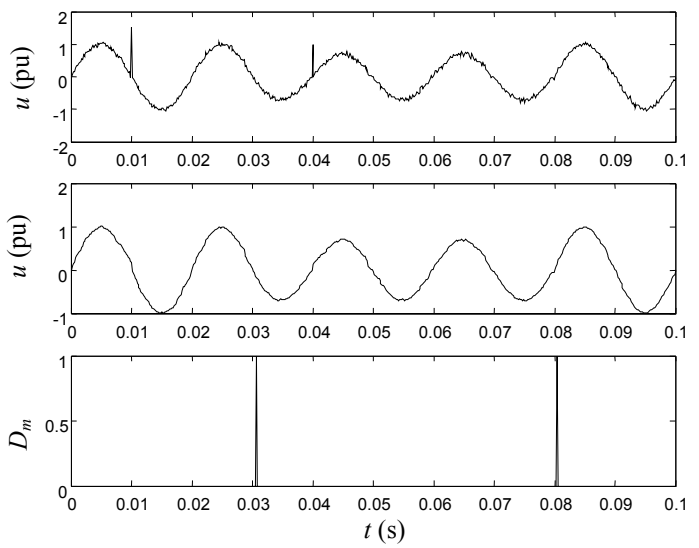

Fig. 7. Voltage sag signal with noise and its filter and time location results

\section{B. Voltage swells}

Voltage swells are referred to voltage magnitude beyond $10 \%$ of the rated value (in the range of 1.1 1.8pu) and lasting for $10 \mathrm{~ms} \sim 1 \mathrm{~min}$, generally caused by single phase short-circuit or tripping off, which usually make the equipments malfunction. Fig. 8 shows the detection results for a voltage

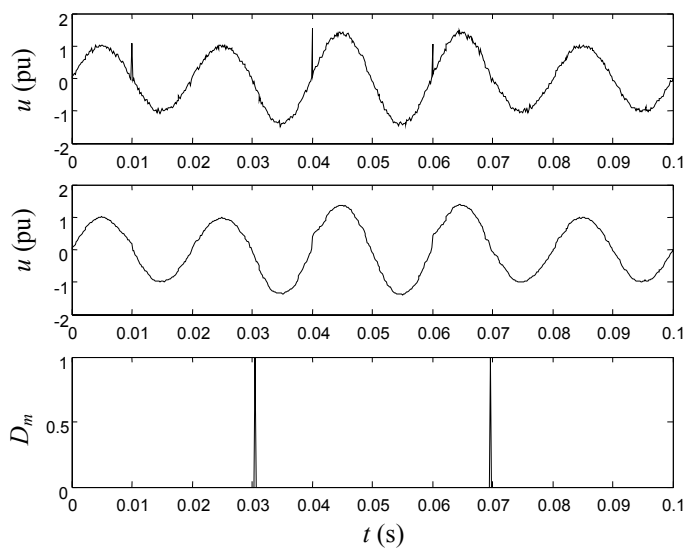

Fig. 8. Voltage swell signal with noise and its filter and time location results curve with a swell lasting for $50 \mathrm{~ms}$ and the rising magnitude by $30 \%$, and white noise variance 0.04 together with two pulses with magnitudes $1.5 \mathrm{pu}$ and $1 \mathrm{pu}$, respectively.

\section{Harmonic Distortions}

Harmonic distortions are generally caused by nonlinear power load. With harmonic distortions increasing, electrical equipments' lifetime is shortening, power losses are enlarging, relay protection equipments may occur misoperation and communication could be disturbed. Fig.9 demonstrates a voltage harmonic distortion signal lasting for 2 periods, and the corresponding filtering and detection results. Wherein white noise variance is 0.09 together with two pulses whose magnitudes are $1.5 \mathrm{pu}$ and $1 \mathrm{pu}$.
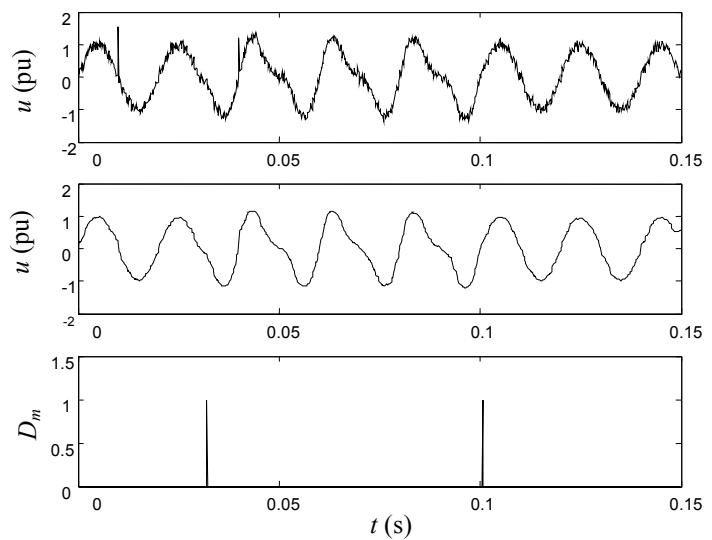

Fig. 9. Harmonic signal with noise and its filtering and time location

The simulation results show that smaller the radius of the semicircular SE is, more better the filter effect is. Trigonal SEs with different length and size give different filtering results corresponding to diverse disturbance types. We need select the most appropriate trigonal SE according to specific disturbance type. The multi-SE composite filter is also affected by each of these elements. If single SE's SNR is high, then the compound filter consisted of multiple these SEs would achieve much better effect. Table II shows the SNR comparisons between the four kinds of SEs and the multipleSE relevant to the above disturbances.

TABLE II

SNR COMPARISONS OF DIFFERENT DISTURBANCE TYPES (dB)

\begin{tabular}{ccccccc}
\hline $\begin{array}{c}\text { Disturbance } \\
\text { signal }\end{array}$ & $\begin{array}{c}\text { Original } \\
\text { signal }\end{array}$ & SEM 1 & SEM 2 & TRI 1 & TRI 2 & MUL \\
\hline Voltage sag & 17.06 & 22.44 & 24.09 & 24.94 & 25.28 & 25.38 \\
Voltage swell & 19.71 & 22.48 & 25.34 & 26.18 & 26.04 & 26.30 \\
HAR & 16.75 & 21.87 & 23.14 & 23.35 & 23.17 & 23.47 \\
\hline
\end{tabular}

HAR means harmonic distortion signal. SEM means filtered by semicircular SE. TRI means filtered by trigonal SE. MUL means filtered by multiple SEs.

\section{Harmonic Distortions and Voltage Swell}

Fig.10 plots a signal mixed with voltage swell, which lasts for $100 \mathrm{~ms}$ and the magnitude rising by $40 \%$, and a harmonic distortion lasting for 2 periods, and the corresponding time location result. Wherein white noise variance is 0.04 together with three pulses with magnitudes $1.5 \mathrm{pu}, 1 \mathrm{pu}$ and $1 \mathrm{pu}$, respectively. 


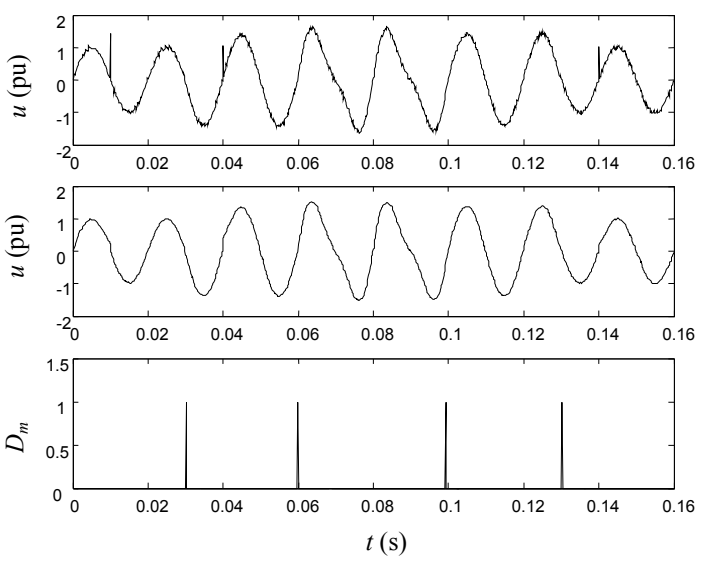

Fig. 10. Filtering and time location results for a mixed signal with harmonic distortion and voltage swell and noise

Simulation results show that using the grille fractal dimension could achieve better effect in locating the start and end time of the disturbance. In attempt of getting $D_{m}$, the threshold has very important role in the accuracy of the detection. It should be adjusted through multiple tests. Table III shows the comparisons between the simulated time and the located time for different disturbances.

TABLE III

TIME LOCATION COMPARISONS OF DIFFERENT DISTURBANCE TYPES (s)

\begin{tabular}{|c|c|c|c|c|}
\hline Disturbance signal & $\begin{array}{c}\text { Simulated } \\
\text { start time }\end{array}$ & $\begin{array}{c}\text { Simulated } \\
\text { restore time }\end{array}$ & $\begin{array}{c}\text { Located } \\
\text { start time }\end{array}$ & $\begin{array}{l}\text { Located } \\
\text { restore time }\end{array}$ \\
\hline Volt & 0.03 & 0.07 & 0.0306 & 0.0698 \\
\hline Voltage & 0 & 0.0 & & 06 \\
\hline Harmonic distortion & 0.03 & 0.1 & 0.0309 & 0.1005 \\
\hline cus & 0.03 & 0.13 & 0.0302 & 0.1304 \\
\hline harmonic & 0.06 & 0.1 & 0.0603 & 0.0993 \\
\hline
\end{tabular}

\section{CONCLUSIONS}

A novel multiple SE composite filter approach is proposed in this paper based on morphological filter technique that can filter effectively several kinds of noise while keep the main feature of the sampling signal. Furthermore, based on the theory of grille fractal dimension, a simplified algorithm based on grille singularity analysis is proposed to detect the start and end time of disturbance. This algorithm is undertaken by calculating the number of grille covered the transient disturbance signals, and comparing its change to locate the time of disturbance occurrence accurately. The numerical tests show the proposed location algorithm has good performances in calculation speed and accuracy.

\section{REFERENCES}

[1] J. Serra, "Morphological filtering: an overview," Signal Processing, vol. 38, no. 1, pp. 3-11, Jan. 1994.

[2] J. Serra, Image Analysis and Mathematical Morphology, New York: Academic, 1982, pp.12-58.

[3] P. Maragos, R W. Schafer, "Morphological systems for multidimensional signal processing," Proceedings of the IEEE, vol. 78, no. 4, pp. 690-710, April 1990.

[4] P. Maragos, "Differential morphology: multiscale image dynamics, maxmin difference equations, and slope transforms," IEEE International Conference on Image Processing, vol. 2, Austin (TX), pp. 545-549, 1316, Nov. 1994.
[5] M. H. Sedaaghi, Q. H. Wu, "Real-time implementation of grey-scale morphological operators, " Electronics Letters, vol. 33, no. 21, pp.176163, Oct. 1997.

[6] M. H. Sedaaghi, Q. H. Wu, "Weighted morphological filter," Electronics Letters, vol. 34, no. 16, pp. 1566-67, Aug. 1998.

[7] Wei Yue, Pei Liu, "Detection of power quality disturbances based on mathematical morphology filter," Automation of Electric Power Systems, vol. 26, no. 7, pp. 13-17, April 2002.

[8] Xiangning Lin, Pei Liu, "A novel integrated morphology-wavelet filter algorithm for ultra-high speed protection of power systems," Proceedings of the CSEE, vol. 22, no. 9, pp. 19-24, Sep. 2002.

[9] T. C. Harsey, M. H. Jensen, L P. Kadanoff, "Fractal measures and their singularities: the characterization of strange sets," Physical Review A, vol.32, no. 2, pp. 1141-1151, 1986.

[10] D. Jaggard, X. Sun, "Fractal surface scattering: a generalized Rayleigh solution," Appl Phs, vol. 68, no. 11, pp. 5356-5462, 1990.

[11] Lo T., H. Leung, "Fractal characterization of seascattered signals and detection of sea surface targets," IEEE Proc-F Radion and Signal Processing, vol. 140, no. 4, pp. 243-249, 1993.

[12] A. V. Mamishev, B. D. Russell, C. L. Denner, "Analysis of high impedance faults using fractal techniques," IEEE Power Industry Computer Applications Conference, pp.401-416, June 1995.

[13] Chunhui Zhao, Shenghe Sun, "A class of parallel complex morphological filters with multiple structuring elements," Journal of Harbin Institute of Technology, vol. 29, no. 2, pp.64-67, April 1997.

[14] Ruina Zhao, Zefeng Qi, Yunping Chen, Luojiang Qian, "Detection of power quality disturbances based on short-duration grille fractal dimension," Relay, vol. 29, no. 11, pp. 21-23, Nov. 2001.

[15] Xiaofei Zhang, Dazhuan Xu, Zefeng Qi, Ruina Zhao, "Detection of Singular Signal Based on Fractal Technique," Journal of Nanjing University of Aeronautics \& Astronautics, vol.35, no.4, pp. 404-408, Aug. 2003.

[16] Qi Cui, Image Process and Analysis: Mathematical Morphology Technique and Application, Beijing: Science Press, 2002.

[17] Heping Xie, Yongping Zhang, Fractal Geometry: Mathematic Foundation and Application, Chongqing: Chongqing University Press, 1991.

[18] D. D. Sabin., A. Sundaram, "Quality enhances reliability," IEEE Spectrum, vol. 33, no. 2, pp.34-41, Feb. 1996.

\section{BIOGRAPHIES}

Gengyin Li (M'03) was born in Hebei Province, China, on May 18, 1964. He received the B.S., M.S. and Ph.D. degrees, all in Electrical Engineering, from North China Electric Power University (NCEPU) in 1984, 1987 and 1996, respectively. Since 1987, Dr. Li has been with the Department of Electrical Engineering at NCEPU, where he is currently a professor and deputy head of the Department. His research interests include electricity markets, power quality, analysis and control of power systems, and new transmission and distribution technologies.

Ming Zhou received the B.S. and M.S. degrees in Electrical Engineering from North China Electric Power University (NCEPU) in 1989 and 1992, respectively. Now she is pursuing Ph. D. degree at NCEPU. Since 1992, Ms. Zhou has been with the Department of Electrical Engineering at NCEPU, where she is currently an associate professor. Her areas of interest include AI applications to power systems, electricity markets, and power system operation and management.

Yan Luo was born in Hebei Province, China, on April 12, 1980. She received the B.S. degree in power system and its automation from North China Electric Power University (NCEPU) in 2002. Since 2002, Miss Luo has been a postgraduate in Electrical Engineering at NCEPU. Her area of interest is power quality.

Yixin Ni (SM'94) received her B. Eng., M. Eng., and Dr. Eng. degrees all in electrical engineering, from Tsinghua University, China. She was a former Professor and Director of National Power System Lab, Tsinghua University and now is with the University of Hong Kong. Her interests are power system stability and control, FACTS, AI applications in power systems and power markets. 